Published in final edited form as:

Biochemistry. 2019 February 12; 58(6): 848-857. doi:10.1021/acs.biochem.8b01167.

\title{
Probing the Electrostatic and Steric Requirements for Substrate Binding in Human Platelet-type 12-Lipoxygenase
}

\author{
Ansari Mukhtar Aleem ${ }^{\dagger,+}$, Wan-Chen Tsai ${ }^{\dagger}$, Jennyfer Tena ${ }^{\dagger, \Phi}$, Gabriella Alvarez, Joshua \\ Deschamps $^{\dagger}, \S$, Chakrapani Kalyanaraman ${ }^{\ddagger}$, Matthew P. Jacobson ${ }^{\ddagger}$, Theodore Holman ${ }^{\dagger,}$, \\ tDepartment of Chemistry and Biochemistry, University of California Santa Cruz, Santa Cruz, CA \\ 95064, United States \\ FDepartment of Pharmaceutical Chemistry, School of Pharmacy, University of California San \\ Francisco, San Francisco, CA 94143, United States
}

${ }^{+}$Current location: A. B. Hancock, Jr. Memorial Laboratory for Cancer Research, Department of Biochemistry, Vanderbilt University, Nashville, TN 37232, USA

\section{Abstract}

Human platelet hALOX12 (hALOX12 or h12-LOX) has been implicated in a variety of human diseases. The present study investigates the active site of hALOX12 to more thoroughly understand how it positions the substrate and achieves nearly perfect regio- and stereo-specificities (i.e., $100+/-5 \%$ of the 12 (S)-hydroperoxide product), utilizing site-directed mutagenesis. Specifically, we have determined that Arg402 is not as important in substrate binding as previously seen for hALOX15, but that His596 may play a role in anchoring the carboxy terminal of the arachidonic acid during catalysis. In addition, Phe 414 creates a pi-stacking interaction with a double bond of arachidonic acid $\left(\Delta^{11}\right)$, and Ala417/Val418 define the bottom of the cavity. However, the influence of Ala417/Val418 on the profile is markedly less for hALOX12 than that seen in hALOX15. Mutating these two residues to larger amino acids (Ala417Ile/Val418Met), only increased the generation of $15-\mathrm{HpETE}$ by $24+/-2 \%$, but conversely smaller residues at these positions converted hALOX15 to almost 100\% hALOX12 reactivity (JBC, 1996, 271, 2541225418). However, we were able to increase 15 -HpETE to $46+/-3 \%$ by restricting the width of the active site with the Ala417Ile/Val418Met/Ser594Thr mutation, indicating both depth and width of the active site are important. Finally, residue Leu407 is shown to play a critical role in positioning the substrate correctly, as seen by the increase of 15 -HpETE to $21+/-1 \%$ for the single Leu407Gly mutant. These results outline critical differences between the active site requirements of hALOX12 relative to hALOX15, and explain both their product specificity and inhibitory differences.

*Corresponding Author: Tel.: +1 831459 5884; fax: +1 831459 2935. holman@ucsc.edu.

Current Address: Department of Chemistry, University of California Davis, Davis, CA 95616

§Current Address: Two Pore Guys Inc., 2161 Delaware Ave Ste B, Santa Cruz, CA 95060 


\section{INTRODUCTION}

Lipoxygenases $(\mathrm{LOXs})^{1}$ are ubiquitous non-heme iron-containing dioxygenases that catalyze the oxidation of polyunsaturated fatty acids (PUFAs) with a cis,cis-1,4-pentadiene moeity. ${ }^{2,3}$ Human LOXs are classified into three major categories, 5-LOX, ${ }^{4} 12-\mathrm{LOX},{ }^{5}$ and $15-\mathrm{LOX}^{6}$, designating which carbon in arachidonic acid (AA) is oxygenated to produce their respective hydroperoxyeicosatetraenoic acid (HpETE) product. The enzymes can be further classified based on the chirality of oxygen insertion, i.e., 'R' or ' $S$ ' LOXs. The HpETE products are subsequently reduced by glutathione peroxidase to form the hydroxyeicosatetraenoic acid (HETE). ${ }^{7-12}$ Accordingly, 12-lipoxygenase catalyzes the transformation of arachidonic acid into 12-HpETE. In humans, two 12-lipoxygenase isozymes are expressed, platelet-type 12(S)-Lipoxygenase (hALOX12 or h12-LOX) and epithelial-type 12(R)-Lipoxygenase (hALOX12B or 12R-LOX). ${ }^{13,14}$ hALOX12 makes the $\mathrm{S}$-product and is primarily expressed in platelets, whereas hALOX12B is primarily expressed in epithelial tissue and makes the R-product. hALOX12 has garnered the majority of scientific attention because of its implication in a variety of human diseases, such as skin inflammation, ${ }^{15}$ certain cancers (i.e. breast, ${ }^{16,17}$ pancreatic $^{18}$ and prostate ${ }^{19}$ ), blood coagulation, ${ }^{20}$ platelet activation ${ }^{21,22}$ and diabetes. ${ }^{23}$

One of the critical properties of LOXs is their ability to oxygenate fatty acids both regio- and stereo-specifically. The structural basis for this product specificity has been investigated extensively for human reticulocyte 15-lipoxygenase-1 (hALOX15 or h15-LOX-1). Sigal and coworkers determined that F414 formed a pi-pi stacking interaction with the $\Delta^{11}$-double bond of AA, while I417/M418 defined the bottom of the active site cavity for hALOX15, which positions the appropriate carbon for oxygenation. ${ }^{24,25}$ This work was expanded by Kuhn and coworkers, who established that F353 in rabbit reticulocyte ALOX15 (rALOX15 or $\mathrm{r} 15-\mathrm{LOX}-1$ ) also played a critical role in defining the overall size/shape of the active site, and thus the specificity for $\mathrm{C}-15$ versus $\mathrm{C}-12$ oxidation of AA (F352 in hALOX15). ${ }^{26,27}$ These data led to the "triad" concept for rALOX15, wherein F353, I418 and I593, positioned in the deepest portion of the substrate-binding pocket, were critical for positional specificity (F352, I417 and I592 in hALOX15). ${ }^{28}$ Vogel et al. concluded that this hypothesis could explain the positional specificity of several mammalian ALOX15 enzymes, specifically those from rabbit, human, M. mulatta, P. pygmaeus and mouse. ${ }^{28}$ However, the specificity of hALOX12 was only partially explained by the triad hypothesis, and for human epithelial ALOX15B (hALOX15B or h15-LOX-2) and hALOX12B, the triad hypothesis did not apply. ${ }^{28}$ It has also been hypothesized that proper alignment of the substrates in LOX active sites additionally requires specific interactions with the carboxylate of the fatty acid. R402 has been proposed to play this role in hALOX $15,{ }^{25}$ but the effect of mutating this amino acid on enzyme kinetics could also be partially explained by de-stabilization of the tertiary structure. ${ }^{29}$ Overall, a three-point interaction mechanism is proposed for hALOX15 to align the substrate in the active site so that the iron abstracts a hydrogen from the appropriate bisallylic carbon: (i) hydrophobic interaction of the methyl end of the substrate with the hydrophobic side chains of the bottom of the active site pocket, F352, I417 and I592; (ii) ionic interaction of the carboxylate end of the fatty acid with R402; ${ }^{25}$ and (iii) $\pi-\pi$ interaction between the fatty acid double bond $\left(\Delta^{11}\right)$ and F414. ${ }^{25}$ 
The structural basis for product selectivity in hALOX12 has been less extensively studied, and previous mutagenesis studies indicate the positional specificity is more complicated than for human/rabbit ALOX15. For porcine leukocyte ALOX15 (pALOX15 or p12-LOX), a double mutation, V418I/V419M, was sufficient to produce $85 \%$ 15-HpETE, versus $10 \%$ for the wild-type enzyme. ${ }^{30}$ However, pALOX15 is more closely related to hALOX15 than hALOX12. For hALOX12, the factors governing positional specificity are not as straightforward. In particular, hALOX12 generates insignificant quantities of 15-HpETE, and the double mutant, A417I/V418M (I417/M418 for hALOX15 and V418/V419 for pALOX15) increased the 15-HpETE percentage to only $14 \% .{ }^{28} \mathrm{~K} 416$ was also proposed to be a specificity determinant but the triple mutant K416Q/A417I/V418M only increased 15HpETE to $20 \%$ of the total. ${ }^{31}$ The substitution of the remaining amino acid variants between positions 398-429 in hALOX12 to the corresponding hALOX15 amino acids (T401V/ Q406G/I408V/G411M-/K416Q/A417I/V418M) increased 15-HpETE to 66\%, however, the enzyme activity was reduced to $25 \%$ of the wt-hALOX12 activity, indicating extensive structural changes. ${ }^{31}$ Other aspects of the three-point interaction hypothesis, including the positioning of the carboxylate (R402) and possible pi-pi interactions (F414), were not previously tested for hALOX12.

In the current work, we expand our investigations of the size and shape of the active site of hALOX12, as well as probe the role of R402 and F414. This work refines the multi-point interaction properties between AA and hALOX12, and determine that the hALOX12substrate interactions are similar but not analogous to the previously described interactions observed in hALOX15.

\section{MATERIAL AND METHODS}

\section{Chemicals.}

All the fatty acids used in this study were purchased from Nu-Chek Prep, Inc. (MN, USA). All other solvents and chemicals were of reagent grade or better and were used as purchased without further purification.

\section{Homology Model of hALOX12 and docking of arachidonic acid.}

A crystal structure of human ALOX12 is available in the Protein Data Bank (pdb id: 3d31, resolution 2.6 ), however, this structure is not suitable for the present modeling due to several missing residues. For instance, helix a 11, located at the entrance of the active site in rabbit ALOX15 and porcine ALOX15 structures, is missing in the hALOX12 structure. Also, helix a 2, positioned parallel to the helix a 11 in rabbit ALOX15 and porcine ALOX15 structures, is re-oriented horizontally above the active site entrance in the hALOX12 structure (structural superposition is shown in Figure S1 of Supplementary Information). Therefore, the active site is fully exposed to the solvent. Further, the C-terminal residue, I663, whose carboxylate coordinates to the active site ferric ion, is missing in the hALOX12 structure. Finally, McGovern et. al. have shown that structure-based virtual screening calculations performed better if the structure had a ligand bound in the active site, ${ }^{32}$ but the hALOX12 structure does not contain a ligand in the active site. However, the structure of porcine leukocyte ALOX15, a close homolog of hALOX12, (pdb is: 3rde, 66\% sequence 
identity), contains a substrate-mimetic, inhibitor bound in the active site. In addition, its crystal structure resolution is $1.9 \AA$, better than that of the hALOX12 structure. Therefore, we decided to build a homology model of hALOX12 based on the porcine hALOX15 structure. The homology model of hALOX12 (Uniprot accession P18054) was built using the software PRIME (v4.7, Schrödinger Inc). During homology modeling we retained the metal ion $\left(\mathrm{Fe}^{3+}\right)$, a hydroxide ion coordinated to the metal ion, and the co-crystallized ligand, 3-(4-[(tridec-2-yn-1-yloxy) methyl]phenyl)propanoic acid, from the porcine ALOX15 structure. The hALOX12 model was subsequently energy minimized using Protein Preparation Wizard (Schrödinger Inc). During the protein preparation step, hydrogen atoms were optimized to make better hydrogen bonding interactions and all heavy atoms were relaxed such that they did not move beyond $0.3 \AA$ from their starting position. After the protein preparation step, we deleted the co-crystallized ligand from the template structure, but retained the metal $\left(\mathrm{Fe}^{3+}\right)$ and hydroxide ion.

The AA substrate and a C10-carbanion reaction intermediate were prepared using the Edit/ Build panel (Maestro v11.1.012, Schrödinger Inc), and then energy minimized using LigPrep (v41012, Schrödinger Inc). We set OPLS-2005 atom-type parameters for the reaction intermediate during LigPrep energy minimization. We subsequently used Glide (v7.4, Schrödinger Inc) to dock both compounds using flexible ligand sampling and extra precision (XP) scoring function options. Glide docking consisted of a grid preparation step and a docking step. We used the centroid of the co-crystallized ligand from the template structure to define the binding site.

\section{Site-directed Mutagenesis.}

Guided by the model of hALOX12 and the predicted binding model of the arachidonic acid C10 carbanion reaction intermediate, we chose the following amino acid mutations to test their importance for substrate positioning: R402L, L407G, L407A, F414L, F414W, A417I, V418M, A417I/V418M, A417I/V418M/S594T and H596L. The numbering in the text refers to UniProt accession number P18054 for the hALOX12 sequence without 6XHis-tag and with the N-terminal methionine assigned as amino acid number one. Online QuikChange Primer Design tool (http://www.genomics.agilent.com/primerDesignProgram.jsp) from Agilent Technologies (CA, USA) was used to design the primers for all the mutants of hALOX12. The mutations were introduced using a QuikChange ${ }^{\circledR} I I$ XL site-directed mutagenesis kit from Agilent Technologies using the provided protocol. The mutations were confirmed by sequencing the hALOX12 insert in the pFastBac1 shuttle vector (Eurofins Genomics, KY, USA).

\section{Protein Expression and Purification.}

The expression and purification of hALOX12 and all mutant enzymes used in this study were performed as previously described. ${ }^{33}$ The wild-type hALOX12 (wt-hALOX12) enzyme and its mutants were expressed as fusion proteins with a 6-His tag on the Nterminus and were purified by affinity nickel-iminodiacetic acid agarose using a FPLC (Biorad). The entire purification process was performed at $4{ }^{\circ} \mathrm{C}$. The purity of all proteins was greater than $90 \%$, as determined by SDS-PAGE analysis and the oligomeric state similar to wt-hALOX12, as determined by SEC. 


\section{Determination of Iron Content using ICP-MS.}

The iron content of wt-hALOX12 and all the mutant enzymes was determined on a Thermo Element XR inductively coupled plasma mass spectrometer (ICP-MS). Cobalt (EDTA) was used as an internal standard. Iron concentrations were compared with standardized iron solutions and all the Kinetic data were normalized to the iron content. Protein concentrations were determined by a Bradford assay, with bovine serum albumin (BSA) as protein standard.

\section{Steady-state Kinetics.}

hALOX12 and all the mutant protein enzymatic rates were determined by following the formation of the conjugated diene product, 12(S)-HpETE, $\left(\varepsilon=27,000 \mathrm{M}^{-1} \mathrm{~cm}^{-1}\right)$ at $234 \mathrm{~nm}$ with Perkin-Elmer Lambda $40 \mathrm{UV} /$ Vis spectrophotometer. The reactions were done in triplicate and started by adding approximately $40 \mathrm{nM}$ enzyme to a $2 \mathrm{~mL}$ reaction mixture containing 1 to $20 \mu \mathrm{M} \mathrm{AA}$, in $25 \mathrm{mM}$ HEPES buffer ( $\mathrm{pH} 8.00$ ), at room temperature $\left(23^{\circ} \mathrm{C}\right.$ ), with constant stirring. Triton X-100 ( $0.01 \%$ by wt) was added to reduce substrate inhibition, of which minimal inhibition was observed up to $20 \mu \mathrm{M}$ AA. Kinetic data were obtained by recording initial enzymatic rates at each substrate concentration and then fitting them to the Michaelis-Menten equation using the KaleidaGraph (Synergy) program to determine $\mathrm{k}_{\text {cat }}$ and $\mathrm{k}_{\mathrm{cat}} / \mathrm{K}_{\mathrm{M}}$ values.

\section{LC/MS Analysis of Enzymatic Products.}

To determine the products formed, wt-hALOX12 and the mutant enzymes were added to a solution of $25 \mathrm{mM}$ HEPES buffer (pH 7.5) and $10 \mu \mathrm{M}$ of a fatty acid substrate (arachidonic acid, docosahexaenoic acid, eicosadienoic acid, gamma linoleic acid, linoleic acid) for 10 minutes, in triplicate. The reactions were quenched with $1 \%$ glacial acetic acid and extracted three times with dichloromethane (DCM). The products were then reduced with trimethylphosphite and evaporated under a stream of $\mathrm{N} 2$ gas. The reaction products were reconstituted in methanol and analyzed via LC-MS/MS. Chromatographic separation was performed using a C18 Synergi $(4 \mu \mathrm{M})$ Hydro-RP 80 Angstrom LC column $(150$ x 2mm). The injection volume was $20 \mu \mathrm{L}$. The chromatography system was coupled to a ThermoElectron LTQ LC-MS/MS for mass analysis. All analyses were performed in negative ionization mode at the normal resolution setting. The 2 mobile phases (A and B) consisted of water with $0.1 \%(\mathrm{v} / \mathrm{v})$ formic acid and acetonitrile with $0.1 \%$ formic acid, respectively. The protocol ran $60 \%$ mobile phase $\mathrm{A}$ and $40 \%$ mobile phase B initially, then at 30 minutes decreased mobile phase A to $55 \%$ and increased mobile phase B to $45 \%$, and finally gradually decreased the percentage of mobile phase $\mathrm{A}$ and increased the percentage of mobile phase B until it reached $25 \%$ mobile phase A and $75 \%$ mobile phase B at 60 minutes. The flow rate was $200 \mu \mathrm{L} / \mathrm{min}$. The products were ionized by electrospray ionization. MS2 was performed in a targeted manner with a mass of 319.5 (for HETE detection). Thermo PDA Plus UV detectors were used. Products were identified by matching retention times, fragmentation and UV spectra to known standards. 


\section{RESULTS and DISCUSSION \\ Model of hALOX12 with substrate and reaction intermediate}

To guide the design and interpretation of the mutagenesis studies, we created a homology model of hALOX12, and then used computational docking to predict the binding mode of arachidonic acid (substrate) and a carbanion reaction intermediate (an approximation of the arachidonyl radical). The LOX reaction mechanism involves the abstraction of a hydrogen atom from the bisallylic methylene, thereby producing a free radical at the methylene carbon (i.e. C10). Unfortunately, the force-field used in molecular docking calculations does not support free radical atom-type. Therefore, we modeled the lone-pair electron on C10 as a carbanion for docking purpose. Results using the intermediate were more consistent with available experimental data, similar to earlier work by Hermann et al., who showed that docking high-energy reaction intermediates of substrate molecules was better in identifying substrates than docking ground-state substrate molecules alone in a large metabolite library docking study. ${ }^{34}$ An extra-precision (XP) docking score of -4.2 was obtained and its docking pose is shown in Figure 1. The carbanion locks the substrate into a position consistent with the characterized enzyme mechanism, in which the ferric-hydroxide abstracts the hydrogen atom from $\mathrm{C} 10$, generating a pentadienyl radical. Specifically, the carbanion on $\mathrm{C} 10$ is $4.9 \AA$ from the metal ion $\left(\mathrm{Fe}^{3+}\right)$ and $3.0 \AA$ to the hydrogen atom of the hydroxide ion. As expected, the hydrophobic $\mathrm{C} 20$ terminal methyl of AA binds deeply in the hydrophobic pocket created by F352, F414, V418, C559 and L589. The distance between the carbanion intermediate and the side chains of residues that interact with the intermediate are given in the Supporting Information (Table S1). The predicted docking score for AA was -2.3 and it bound outside the catalytic site.

\section{Binding interactions with the carboxylate moiety of arachidonic acid}

Previously, Gan et al. demonstrated that R402 in hALOX15 interacted with the carboxylate moiety of the fatty acid substrate, positioning the substrate in the active site relative to the catalytic iron for hydrogen atom abstraction. ${ }^{25} \mathrm{R} 402$ is conserved in both hALOX15 and hALOX12 (R402), suggesting that it might play a similar role for fatty acid binding in hALOX12. However, the model of hALOX12 with the reaction intermediate predicts that the carboxylic acid of AA makes a hydrogen bonding interaction with H596 (Q595 in hALOX15) and not R402. We therefore mutated both R402 and H596 to test these competing hypotheses.

To interrogate the role of R402, we replaced it with a nonpolar leucine to disrupt the proposed hydrogen bond interaction with the substrate. This hALOX12 mutant, R402L, demonstrated only a 2 -fold decrease in $\mathrm{k}_{\text {cat }} / \mathrm{K}_{\mathrm{M}}$ when compared to wt-hALOX12, $6.3 \pm 0.5$ $\mu \mathrm{M}^{-1} \mathrm{~s}^{-1}$ and $14 \pm 0.9 \mu \mathrm{M}^{-1} \mathrm{~s}^{-1}$, respectively (Table 1). There was also no significant change in the $\mathrm{K}_{\mathrm{M}}$ value of $\mathrm{AA}$, which was $0.89 \pm 0.09 \mu \mathrm{M}$ for R402L and $1.9 \pm 0.2 \mu \mathrm{M}$ for wthALOX12. R402L therefore has an effect on catalysis, but it is not nearly as dramatic as that seen for hALOX15, where $\mathrm{k}_{\text {cat }} / \mathrm{K}_{\mathrm{M}}$ decreased by 136 -fold with the analogous mutation. The R402L mutation also did not significantly impact the positional specificity of hALOX12, as both wt-hALOX12 and R402L yielded approximately 100\% 12-HETE (Table 1), in contrast to previous results on hALOX15, which showed a larger change in product profile. Both the 
kinetic and specificity results suggest that R402 of hALOX12 does not interact with AA as significantly as it does in hALOX15. It should be noted that in Plexaura homomalla (black sea whip coral) $8 R$-LOX (cALOX8), 35 they observe R182 directly interacting with the carboxylate of AA, but the R182L mutant only manifests a 2-fold decrease in $\mathrm{k}_{\mathrm{cat}} / \mathrm{K}_{\mathrm{M}}$, similar to hALOX12. However, they also observe significant substrate inhibition with the R182L mutant, which we do not observe for the hALOX12 R402L mutant. Considering that cALOX8 only shares $30 \%$ sequence identity with hALOX12, it is difficult to compare these two LOX isozymes with respect to this mechanistic detail, but we still cannot completely rule out a substrate binding role for R402 in hALOX12 based on the cALOX8 result.

hALOX12 also accepts docosahexaenoic acid (DHA) as a substrate, with similar reaction kinetics to that of AA (Table 1) and since DHA is 2 carbons longer than AA, it is possible DHA could interact with R402. We therefore tested if R402 might coordinate the carboxylate of DHA, however, no significant change in the activity was observed with DHA between wt-hALOX12 and R402L, further supporting our hypothesis that R402 in hALOX12 does not interact with the carboxylate group of its fatty acid substrates significantly. A minor change was observed in the product distribution which also supports a minimal effect of R402 on catalysis. It should be noted that the percent of 11-HDHA:14HDHA is larger than reported previously with hALOX12 from E. coli., ${ }^{36}$ which is possibly due to the fact that the current work expresses hALOX12 in SF9 cells. We are currently investigating this further.

To test the alternate hypothesis suggested by the computational model, H596 was replaced with a nonpolar leucine (H596L) to disrupt H-bonding with the substrate, but only a relatively small decrease in enzyme activity was observed. Kinetic characterization of H596L under steady-state conditions showed a 2-fold decrease in $\mathrm{k}_{\text {cat }} / \mathrm{K}_{\mathrm{M}}$ relative to wthALOX12, $6.3 \pm 0.4$ and $14 \pm 0.9$, respectively, and 3-fold decrease in $\mathrm{k}_{\text {cat }}\left(8.6 \pm 0.7 \mathrm{~s}^{-1}\right)$ relative to wt-hALOX12 $\left(28 \pm 0.9 \mathrm{~s}^{-1}\right)$ (Table 1). However, more significant changes were observed in positional specificity, with H596L producing both 12-HETE and 15-HETE, in a ratio of $\sim 3: 1$, along with a small amount of 11 -HETE $(5+/-1 \%)$, while wt-hALOX12 produced 100+/- 5\% 12-HETE. We hypothesize that in H596L, the carboxylate group of AA now interacts with R402, which could explain the production of 15-HETE upon H596L mutation. In order to test this hypothesis, we docked C13-carbanion intermediate of AA to hALOX12 with H596L mutation, and the carboxylate group can indeed hydrogen bond to $\mathrm{R} 402$, positioning the reactive carbon atom, $\mathrm{C} 13,5.0 \AA$ away from the metal ion (please see supplemental information, Figure S2). These results suggest that H596 in wt-hALOX12 may contribute to properly positioning AA for catalysis, possibly through its interaction with the carboxylate moiety of AA.

\section{$\pi-\pi$ interactions of AA double bonds with aromatic amino acids of hALOX12}

Based on their model of substrate binding in hALOX15, Gan et al. identified F414 as an important substrate binding determinant, ${ }^{25}$ proposing that the $\Delta^{11}$-double bond of AA participated in a $\pi-\pi$ interaction with the aromatic side chain. We mutated the corresponding amino acid in hALOX12, F414L, resulting in a 12 -fold decrease in the $\mathrm{k}_{\text {cat }} / \mathrm{K}_{\mathrm{M}}$, and a 16-fold decrease in $\mathrm{k}_{\text {cat }}$ (Table 2). In contrast, $\mathrm{F} 414 \mathrm{~W}$ resulted in a more 
modest reduction in catalytic activity, manifesting a 3.5-fold decrease in $\mathrm{k}_{\mathrm{cat}} / \mathrm{K}_{\mathrm{M}}$ and a 1.8fold decrease in $\mathrm{k}_{\text {cat }}$, relative to wild-type. These data suggest that the side chain aromaticity at position 414 is important for binding, consistent with F414 forming a $\pi-\pi$ interaction with AA.

Having established the importance of F414, we investigated which specific double bond in AA interacted with F414. The docking model predicted F414 interacted with either $\Delta^{11}$ or $\Delta^{14}$ so we measured the catalytic rates of mead acid (5Z,8Z,11Z-eicosatrienoic acid) with wt-hALOX12 and the two mutant hALOX12s, F414L and F414W. Mead acid has the same 20-carbon length as AA, but only has three double bonds, missing the last $\Delta^{14}$ double bond. It is hypothesized that if F414 interacts with the $\Delta^{14}$ double bond, then wt-hALOX12, F414L and F414W would all show a decreased activity with mead acid. However, if the interaction was with the $\Delta^{11}$ double bond, then the activity of these three LOXs would show the same pattern as seen with AA. As seen in Table 3, the catalytic activity with mead acid follows the same pattern as seen with AA, except that the catalytic efficiency actually increases in F414W relative to wt-hALOX12. We therefore conclude that the docking model and the AA and mead acid kinetic data are consistent with a model in which the aromatic side chain of F414 in hALOX12 $\pi-\pi$ stacks with the $\Delta^{11}$-double bond of AA, similar to that seen for hALOX15. This interaction could facilitate the formation of an allyl radical by delocalizing the $\pi$-electrons, as suggested by earlier studies on hALOX15 and soybean LOX-1. ${ }^{25,}{ }^{37}$. It should be noted that removal of $\Delta^{11}$ from the substrate eliminates all activity of hALOX12 since the activated methylene is lost.

\section{Interaction of the fatty acid tail with the hydrophobic bottom of the active site.}

The importance of the volume of the hydrophobic binding site for the lipid tail has been well established as an important factor in determining the remarkable specificity of lipoxygenases for fatty acid oxygenation. ${ }^{38}$ Mutagenesis studies on various ALOX15 isoforms led to the development of a hypothesis to explain its product specificity, ${ }^{26,27,39}$ which postulates that F352, I417, M418 and 592 (hALOX15 numbering), which lie at the bottom of the catalytic cavity, regulate the depth of fatty acid penetration and thus the proper positioning of the substrate for catalysis. Similar studies for hALOX12, focusing on A417 and V418 at the bottom of the hydrophobic pocket (corresponding to the larger amino acids I417 and M418 in hALOX15), have been less conclusive. V418M resulted in no significant change in positional specificity, ${ }^{28,31}$ while A417I and the double mutant A417M/V418M only altered the positional specificity slightly for hALOX12, with 15\% of 15-HpETE being produced. In the current work, we observed somewhat larger changes in product distribution for the double mutant, A417I/V418M, resulting in 24+/- 2\% 15-HpETE and a small amount of 11HpETE $(0.5+/-0.1 \%)$, as shown in Table 4 . Nonetheless, the overall percent change in positional specificity of these hALOX12 mutants is lower than that seen for mutations in analogous positions for ALOX15s. For example, a single mutation of hALOX15 (I418A) increased the yield of 12-HETE from $14 \%$ for wt-hALOX15 to $94 \%$ for the I418A mutant. ${ }^{28}$

We therefore postulated that the hALOX12 active site was wider than that in hALOX15 and thus could allow for additional space for substrate positioning. The residue, S594, was selected for mutation because its corresponding position in hALOX15 is occupied by a 
slightly bulkier residue threonine, which would make the active site more narrow in hALOX15. We investigated this possibility by creating a triple mutant (A417I/V418M/ S594T) that makes the base of the cavity not only smaller and shallower (A417I and V418M) but also narrower (S594T), as seen in Figure 1. This change results in an increase in 15-HpETE generated to $46+/-3 \%$ for A417I/V418M/S594T, as compared to $23+/-1 \%$ for A417I/V418M.

Considering that A417I/V418M affected the product ratio of AA catalysis, we subsequently investigated the effect these mutations would have on fatty acids with varied length and unsaturation. The substrates utilized were the C-20 fatty acid, 11Z, 14Z-eicosadienoic acid (EDA) and the C-18 fatty acids, 9Z, 12Z-linoleic acid (LA) and gamma 6Z, 9Z, 12Zlinolenic acid (GLA). Previous studies in our lab have shown that EDA and LA are poor substrates for hALOX12 because the bis-allylic hydrogen is not positioned properly for hydrogen atom abstraction (Table 5) ${ }^{40}$ However, the mutant, A417I/V418M, reacts with both of EDA and LA, effectively shifting the substrate backward into the active site so that the bis-allylic carbon, C-13 for EDA and C-11 for LA, are positioned so the catalytic ferrichydroxide abstracts the proper hydrogen atom. The products generated were as expected, $100 \%$ 15-HpEDA with EDA and 100\% 13-HpODE for LA (Table 5). It should be noted that GLA is a substrate for wt-hALOX12, but its $\mathrm{k}_{\text {cat }} / \mathrm{K}_{\mathrm{M}}$ is very low $\left(\mathrm{k}_{\mathrm{cat}} / \mathrm{K}_{\mathrm{M}}=0.08 \pm 0.01 \mu \mathrm{M}\right.$ ${ }^{-1} \mathrm{~s}^{-1}$ ) and two products are generated, 30+/- 2\% 10-HpOTrE and 70+/- 4\% 13-HpOTrE (Table 5). However, the reactivity of GLA with A417I/V418M increases 32 -fold $\left(\mathrm{K}_{\text {cat }} / \mathrm{K}_{\mathrm{M}}=\right.$ $2.6 \pm 0.10 \mu \mathrm{M}^{-1} \mathrm{~s}^{-1}$ ) relative to the wt-hALOX12 and it produces entirely $13-\mathrm{HpOTrE}$, as shown in the Table 5. It should be noted that the wt-hALOX12 result does not follow the classical interpretation of LOX product formation. One would predict that the main product of wt-hALOX12 from GLA would be 10-HpOTrE, due to the positioning of C9 near the active site iron, however, 13-HpOTrE is the major product. It appears that the shorter length of GLA relative to AA, not only lowers the catalytic efficiency but also affects substrate positioning, by increasing the formation of $13-\mathrm{HpOTrE}$ relative to $10-\mathrm{HpOTrE}$. For all three of these fatty acids, the increase in enzyme activity of the A417I/V418M mutant relative to wt-hALOX12 appears to be due to a repositioning of the fatty acid by restricting the depth of the active site, such that the bis-allylic hydrogen is now positioned properly for abstraction. While this effect is dramatic for these three fatty acids since there is little or no activity with wt-hALOX12, it is comparable to the change in reactivity wt-hALOX12 and A417I/V418M with AA. A417I/V418M produces $24+/-2 \% 15-\mathrm{HpETE}$ from AA and given that its $\mathrm{k}_{\text {cat }} / \mathrm{K}_{\mathrm{M}}$ is $59 \mu \mathrm{M}^{-1} \mathrm{~s}^{-1}$, this translates to an approximate $\mathrm{k}_{\mathrm{cat}} / \mathrm{K}_{\mathrm{M}}$ of $14 \mu \mathrm{M}^{-1} \mathrm{~s}^{-1}$ for producing 15 HpETE, which is of comparable magnitude to the $\mathrm{k}_{\text {cat }} / \mathrm{K}_{\mathrm{M}}$ values A417I/V418M with LA, EDA and GLA.

\section{Critical role of $\mathrm{L} 407$ in substrate positioning}

The model of wt-hALOX12 with AA bound suggests that the bulky hydrophobic side chain of L407 plays a critical role in defining the " $U$ " shape of the binding site and thus properly positioning AA for catalysis. Since L407 is conserved in all lipoxygenases ${ }^{25}$ and is observed at the base of the U-shaped AA in the cALOX8 (8R-LOX) co-structure, ${ }^{35}$ we hypothesized that mutations of L407 would affect catalytic efficiency and possibly positional specificity. Specifically, replacing L407 with the much smaller amino acids alanine (L407A) and 
glycine (L407G) was predicted to greatly widen the binding site near the iron, essentially eliminating the characteristic "U" shape (Figure 2). Consistent with this hypothesis, we observed an $\sim 100-$ fold decrease in both $\mathrm{k}_{\text {cat }}$ and $\mathrm{k}_{\text {cat }} / \mathrm{K}_{\mathrm{M}}$ for both of these mutants, when compared to wt-hALOX12 (Table 6), confirming its important role in catalysis. For comparison, we previously mutated this residue in hALOX15 (L407A) and demonstrated a 5 -fold decrease in $\mathrm{k}_{\mathrm{cat}}$ and a 3 -fold decrease in $\mathrm{k}_{\mathrm{cat}} / \mathrm{K}_{\mathrm{M}} \cdot{ }^{41}$ These effects are less than that seen for hALOX12, indicating a reduced effect of L407 in hALOX15.

In addition, widening the active site cavity with L407A caused a slight decrease in positional specificity, with 3+/- 1\% 15-HpETE and 6+/- 1\% 11-HpETE generation. This change in positional specificity increased when the cavity was made even larger with the L407G mutant enzyme, with the percentages of the 15-HpETE and 11-HpETE increasing to $21+/-$ $2 \%$ and $19+/-2 \%$, respectively (Table 6). These results imply that L407 is partly responsible for positioning the $\mathrm{C} 10$ for abstraction, with a smaller residue at position 407 possibly leading to a "looser" substrate binding mode, thus affecting the percent 15-HpETE product synthesized. The triple mutant, L407G/A417I/V418M, increases the percent of 15-HETE even further to $31+/-2 \%$, which is consistent with this hypothesis, since A417I/V418M reduce the length of the cavity and adjust the substrate positioning further, aligning $\mathrm{C} 13$ for hydrogen atom abstraction. Previously, the homologous mutation, L546A, in soybean LOX-1 was shown to affect catalysis significantly, similar to what we observe for L407A. ${ }^{42}$ Klinman and coworkers determined that the decreased rate of L546A was due to an increased cavity size across from the ferric-hydroxide moiety, which altered heavy-atom motions and lead to an increase in the energy of activation for hydrogen atom abstraction. They did not investigate if L546A demonstrated altered product reactivity, but given our results it would be a likely result. It should be noted that the percentage of 11-HETE increases as the cavity increases in size (L407A vs. L407G), but then decreases as the cavity shrinks with the triple mutant (L407G/A417I/V418M), suggesting that the 11-HETE product correlates with a larger cavity. Finally, we generated the 4 residue mutant, A407G/A417I/ V418M/S594T, to increase the 15-HETE percentage even further, but it was inactive, suggesting these four mutations make the enzyme incapable of positioning the hydrogen atom for abstraction. We are currently investigating this result in more detail to understand the substrate binding determinants in more detail.

\section{Substrate positioning in hALOX12 and other lipoxygenases}

The remarkable product specificity achieved by LOX enzymes can be traced to proper positioning of substrates in the active site, specifically, (1) how the carboxylate group of the fatty acid is anchored as the substrate binds in the active site and (2) the depth of the active site. Here we compare residues that co-ordinate to the carboxylate group of AA in hALOX12 to other LOX enzymes. The available crystal structure of rALOX15 does not have a substrate or substrate analog bound in the active site. However, a mutational study showed that R402 from helix a 11 in hALOX15 (equivalent to R403 in rALOX15) anchored the carboxylate group of AA and LA ${ }^{15}$. Structural and mutational studies of CALOX8 $(8 R$ LOX) showed that R182 from helix a 2 hydrogen bonds to the carboxylate group of AA. ${ }^{35,43}$ The pALOX15 crystal structure showed that Q596 from helix a 21 interacted with the carboxylate group of the co-crystallized substrate-analog inhibitor. ${ }^{44}$ Finally, the results of 
this study demonstrate that H596 from helix a 21 plays the role of anchoring carboxylate group of AA in hALOX12.

In Figure 3, we structurally superimpose all of these structures/models, highlighting the carboxylate group anchoring residues. In all cases, the substrate binds in a head-to-tail orientation, with the head (i.e., the carboxylate group) binding at the entrance of the active site and the hydrophobic tail binding at the interior of the protein. However, the side chains coordinating the carboxylate are located on 3 different helices: helix a 11 for rALOX15, helix a 21 for pALOX15, and helix a 2 for cALOX8 ( $8 R$-LOX). These observations, therefore, support the hypothesis that LOX enzymes may have evolved to achieve different regio- and stereo-specificities by selectively positioning the polar residues on different helices near the entrance of active site.

\section{CONCLUSIONS}

Human platelet ALOX12 is a critical enzyme in a variety of human biological processes and therefore understanding its mechanism of action is highly important. In this report, we have performed extensive site-directed mutagenesis and molecular modeling to better understand the binding of fatty acids to the active site of hALOX12. We observed that the conserved R402 in hALOX12 displays minimal interactions with the carboxylate end of AA, as opposed to the large interaction reported for hALOX15. ${ }^{25}$ H596 in hALOX12 appears to be better positioned to interact with the carboxylic acid, but its effects on hALOX12 catalysis are still not as great as that seen for R402 with hALOX15. We also determined that F414 in hALOX12 interacts with the $\Delta^{11}$ double bond of AA, similar to that seen in hALOX15, indicating a common pi-pi stacking determinant for both hALOX12 and hALOX15. ${ }^{25}$ Our results also demonstrate that A417 and V418 are determinants for positional specificity in hALOX12, as observed previously for hALOX15, due to their defining of the cavity depth. ${ }^{28}$ In addition, we observe that S594 also contributes to the positional specificity of hALOX12 by narrowing the cavity and restricting the degrees of freedom the methyl tail of AA can sample, thus increasing 15-HETE production. Finally, L407 appears to provide a restriction in the active site, which constrains the substrate such that the hydrogen from $\mathrm{C} 10$ is abstracted with high efficiency. These findings are summarized in Figure 4, which highlight the key substrate determinants for hALOX12.

\section{Supplementary Material}

Refer to Web version on PubMed Central for supplementary material.

\section{ACKNOWLEDGMENTS}

We are grateful to Angel Baroz and Sahil Khan for their technical help with the experiments. We are also thankful to Steve Perry for useful discussion. MPJ is a consultant to and shareholder of Schrodinger LLC, which licenses the software used in this work.

Funding: NIH: AG047986 


\section{Abbreviations:}

LOX

hALOX15 (h15-LOX-1)

rALOX15 (r15-LOX-1)

hALOX12 (h12-LOX)

pALOX15 (p12-LOX)

cALOX8 (8R-LOX)

PUFA

AA

DHA

EDA

LA

GLA

HETE

HpETE

12-HETE

12-HpETE

11-HETE

15-HETE

15-HpETE

15-HpEDA

13-HODE

13-HpODE

13-HOTrE

13-HpOTrE

10-HpOTrE lipoxygenase

human reticulocyte 15-lipoxygenase-1

rabbit reticulocyte 15-lipoxygenase-1

human platelet 12-lipoxygenase

porcine leukocyte 12-lipoxygenase

Plexaura homomalla (black sea whip coral) 8R-

lipoxygenase

polyunsaturated fatty acids

arachidonic acid

docosahexaenoic acid

11Z, 14Z-eicosadienoic acid

9Z, 12Z-linoleic acid

gamma-6Z, 9Z, 12Z-linolenic acid

hydroxy-eicosatetraenoic acid

hydroperoxy-eicosatetraenoic acid

12-hydroxy-eicosatetraenoic acid

12-hydroperoxy-5Z,8Z,10E,14Z-eicosatetraenoic acid

11-hydroxy-eicosatetraenoic acid

15-hydroxy-eicosatetraenoic acid

15-hydroperoxy-5Z,8Z,10Z,13E-eicosatetraenoic acid

15(S)-hydroperoxy-11Z,13E-eicosadienoic acid

13(S)-hydroxy-9Z,11E-octadecadienoic acid

13(S)-hydroperoxy-9Z,11E-octadecadienoic acid

13(S)-hydroxy-6Z,9Z,11E-octadecatrienoic acid

13(S)-hydroperoxy-6Z,9Z,11E-octadecatrienoic acid

10(S)-hydroperoxy-6Z,8E,12Z-octadecatrienoic acid 


\section{References:}

[1]. Newcomer ME, and Brash AR (2015) The structural basis for specificity in lipoxygenase catalysis, Protein Sci 24, 298-309. [PubMed: 25524168]

[2]. Brash AR (1999) Lipoxygenases: Occurrence, Functions, Catalysis, and Acquisition of Substrate, J. Biol. Chem 274, 23679-23682. [PubMed: 10446122]

[3]. Kuhn H, Saam J, Eibach S, Holzhütter H-G, Ivanov I, and Walther M (2005) Structural biology of mammalian lipoxygenases: Enzymatic consequences of targeted alterations of the protein structure, Biochemical and Biophysical Research Communications 338, 93-101. [PubMed: 16168952]

[4]. Lauretti E, and Pratico D (2018) Novel Key Players in the Development of Tau Neuropathology: Focus on the 5-Lipoxygenase, J Alzheimers Dis 64, S481-S489. [PubMed: 29758943]

[5]. Tersey SA, Bolanis E, Holman TR, Maloney DJ, Nadler JL, and Mirmira RG (2015) Minireview: 12-Lipoxygenase and Islet beta-Cell Dysfunction in Diabetes, Mol Endocrinol 29, 791-800. [PubMed: 25803446]

[6]. Ivanov I, Kuhn H, and Heydeck D (2015) Structural and functional biology of arachidonic acid 15lipoxygenase-1 (ALOX15), Gene 573, 1-32. [PubMed: 26216303]

[7]. Kagan VE, Mao G, Qu F, Angeli JP, Doll S, Croix CS, Dar HH, Liu B, Tyurin VA, Ritov VB, Kapralov AA, Amoscato AA, Jiang J, Anthonymuthu T, Mohammadyani D, Yang Q, Proneth B, Klein-Seetharaman J, Watkins S, Bahar I, Greenberger J, Mallampalli RK, Stockwell BR, Tyurina YY, Conrad M, and Bayir H (2017) Oxidized arachidonic and adrenic PEs navigate cells to ferroptosis, Nat Chem Biol 13, 81-90. [PubMed: 27842066]

[8]. Drefs M, Thomas MN, Guba M, Angele MK, Werner J, Conrad M, Steib CJ, Holdt LM, Andrassy J, Khandoga A, and Rentsch M (2017) Modulation of Glutathione Hemostasis by Inhibition of 12/15-Lipoxygenase Prevents ROS-Mediated Cell Death after Hepatic Ischemia and Reperfusion, Oxid Med Cell Longev 2017, 8325754. [PubMed: 28811867]

[9]. Friedmann Angeli JP, Schneider M, Proneth B, Tyurina YY, Tyurin VA, Hammond VJ, Herbach N, Aichler M, Walch A, Eggenhofer E, Basavarajappa D, Radmark O, Kobayashi S, Seibt T, Beck H, Neff F, Esposito I, Wanke R, Forster H, Yefremova O, Heinrichmeyer M, Bornkamm GW, Geissler EK, Thomas SB, Stockwell BR, O’Donnell VB, Kagan VE, Schick JA, and Conrad M (2014) Inactivation of the ferroptosis regulator Gpx4 triggers acute renal failure in mice, Nat Cell Biol 16, 1180-1191. [PubMed: 25402683]

[10]. Toppo S, Flohe L, Ursini F, Vanin S, and Maiorino M (2009) Catalytic mechanisms and specificities of glutathione peroxidases: variations of a basic scheme, Biochim Biophys Acta 1790, 1486-1500. [PubMed: 19376195]

[11]. Sutherland M, Shankaranarayanan P, Schewe T, and Nigam S (2001) Evidence for the presence of phospholipid hydroperoxide glutathione peroxidase in human platelets: implications for its involvement in the regulatory network of the 12-lipoxygenase pathway of arachidonic acid metabolism, Biochem J 353, 91-100. [PubMed: 11115402]

[12]. Schnurr K, Belkner J, Ursini F, Schewe T, and Kuhn H (1996) The selenoenzyme phospholipid hydroperoxide glutathione peroxidase controls the activity of the 15-lipoxygenase with complex substrates and preserves the specificity of the oxygenation products, J Biol Chem 271, 46534658. [PubMed: 8617728]

[13]. Boeglin WE, Kim RB, and Brash AR (1998) A 12R-lipoxygenase in human skin: mechanistic evidence, molecular cloning, and expression, Proc. Natl. Acad. Sci. U.S.A. 95, 6744-6749. [PubMed: 9618483]

[14]. Chen XS, Brash AR, and Funk CD (1993) Purification and characterization of recombinant histidine-tagged human platelet 12-lipoxygenase expressed in a baculovirus/insect cell system, Eur. J. Biochem 214, 845-852. [PubMed: 8319693]

[15]. Hussain H, Shornick LP, Shannon VR, Wilson JD, Funk CD, Pentland AP, and Holtzman MJ (1994) Epidermis contains platelet-type 12-lipoxygenase that is overexpressed in germinal layer keratinocytes in psoriasis, Am. J. Physiol 266, C243-253. [PubMed: 8304420] 
[16]. Connolly JM, and Rose DP (1998) Enhanced angiogenesis and growth of 12-lipoxygenase genetransfected MCF-7 human breast cancer cells in athymic nude mice, Cancer Lett 132, 107-112. [PubMed: 10397460]

[17]. Natarajan R, and Nadler J (1998) Role of lipoxygenases in breast cancer, Front. Biosci 3, E8188. [PubMed: 9616130]

[18]. Ding XZ, Iversen P, Cluck MW, Knezetic JA, and Adrian TE (1999) Lipoxygenase inhibitors abolish proliferation of human pancreatic cancer cells, Biochemical and Biophysical Research Communications 261, 218-223. [PubMed: 10405349]

[19]. Shappell SB, Olson SJ, Hannah SE, Manning S, Roberts RL, Masumori N, Jisaka M, Boeglin WE, Vader V, Dave DS, Shook MF, Thomas TZ, Funk CD, Brash AR, and Matusik RJ (2003) Elevated expression of 12/15-lipoxygenase and cyclooxygenase-2 in a transgenic mouse model of prostate carcinoma, Cancer Res 63, 2256-2267. [PubMed: 12727848]

[20]. Thomas CP, Morgan LT, Maskrey BH, Murphy RC, Kühn H, Hazen SL, Goodall AH, Hamali HA, Collins PW, and O'Donnell VB (2010) Phospholipid-esterified eicosanoids are generated in agonist-activated human platelets and enhance tissue factor-dependent thrombin generation, The Journal of Biological Chemistry 285, 6891-6903. [PubMed: 20061396]

[21]. Holinstat M, Boutaud O, Apopa PL, Vesci J, Bala M, Oates JA, and Hamm HE (2011) Proteaseactivated receptor signaling in platelets activates cytosolic phospholipase A2a differently for cyclooxygenase-1 and 12-lipoxygenase catalysis, Arterioscler. Thromb. Vasc. Biol 31, 435-442. [PubMed: 21127289]

[22]. Kaur G, Jalagadugula G, Mao G, and Rao AK (2010) RUNX1/core binding factor A2 regulates platelet 12-lipoxygenase gene (ALOX12): studies in human RUNX1 haplodeficiency, Blood 115, 3128-3135. [PubMed: 20181616]

[23]. Ma K, Nunemaker CS, Wu R, Chakrabarti SK, Taylor-Fishwick DA, and Nadler JL (2010) 12Lipoxygenase Products Reduce Insulin Secretion and \{beta\}-Cell Viability in Human Islets, J. Clin. Endocrinol. Metab 95, 887-893. [PubMed: 20089617]

[24]. Sloane DL, Leung R, Barnett J, Craik CS, and Sigal E (1995) Conversion of human 15lipoxygenase to an efficient 12-lipoxygenase: the side-chain geometry of amino acids 417 and 418 determine positional specificity, Protein Eng 8, 275-282. [PubMed: 7479689]

[25]. Gan QF, Browner MF, Sloane DL, and Sigal E (1996) Defining the arachidonic acid binding site of human 15-lipoxygenase. Molecular modeling and mutagenesis, The Journal of Biological Chemistry 271, 25412-25418. [PubMed: 8810309]

[26]. Borngräber S, Kuban RJ, Anton M, and Kühn H (1996) Phenylalanine 353 is a primary determinant for the positional specificity of mammalian 15-lipoxygenases, J. Mol. Biol 264, 1145-1153. [PubMed: 9000636]

[27]. Borngräber S, Kuban RJ, and Kühn H (1999) Sequence determinants for the positional specificity of mammalian and plant lipoxygenases, Adv. Exp. Med. Biol 469, 91-97. [PubMed: 10667315]

[28]. Vogel R, Jansen C, Roffeis J, Reddanna P, Forsell P, Claesson H-E, Kuhn H, and Walther M (2010) Applicability of the triad concept for the positional specificity of mammalian lipoxygenases, The Journal of Biological Chemistry 285, 5369-5376. [PubMed: 20026599]

[29]. Di Venere A, Horn T, Stehling S, Mei G, Masgrau L, González-Lafont A, Kühn H, and Ivanov I (2013) Role of Arg403 for thermostability and catalytic activity of rabbit 12/15-lipoxygenase, Biochim. Biophys. Acta 1831, 1079-1088. [PubMed: 23438511]

[30]. Suzuki H, Kishimoto K, Yoshimoto T, Yamamoto S, Kanai F, Ebina Y, Miyatake A, and Tanabe T (1994) Site-directed mutagenesis studies on the iron-binding domain and the determinant for the substrate oxygenation site of porcine leukocyte arachidonate 12-lipoxygenase, Biochim. Biophys. Acta 1210, 308-316. [PubMed: 8305485]

[31]. Chen XS, and Funk CD (1993) Structure-function properties of human platelet 12-lipoxygenase: chimeric enzyme and in vitro mutagenesis studies, FASEB J 7, 694-701. [PubMed: 8500694]

[32]. McGovern SL, and Shoichet BK (2003) Information decay in molecular docking screens against holo, apo, and modeled conformations of enzymes, J Med Chem 46, 2895-2907. [PubMed: 12825931]

[33]. Amagata T, Whitman S, Johnson TA, Stessman CC, Loo CP, Lobkovsky E, Clardy J, Crews P, and Holman TR (2003) Exploring sponge-derived terpenoids for their potency and selectivity 
against 12-human, 15-human, and 15-soybean lipoxygenases, J. Nat. Prod 66, 230-235. [PubMed: 12608855]

[34]. Hermann JC, Ghanem E, Li Y, Raushel FM, Irwin JJ, and Shoichet BK (2006) Predicting substrates by docking high-energy intermediates to enzyme structures, J Am Chem Soc 128, 15882-15891. [PubMed: 17147401]

[35]. Neau DB, Bender G, Boeglin WE, Bartlett SG, Brash AR, and Newcomer ME (2014) Crystal structure of a lipoxygenase in complex with substrate: the arachidonic acid-binding site of $8 \mathrm{R}$ lipoxygenase, J Biol Chem 289, 31905-31913. [PubMed: 25231982]

[36]. Kutzner L, Goloshchapova K, Heydeck D, Stehling S, Kuhn H, and Schebb NH (2017) Mammalian ALOX15 orthologs exhibit pronounced dual positional specificity with docosahexaenoic acid, Biochim Biophys Acta Mol Cell Biol Lipids 1862, 666-675. [PubMed: 28400162]

[37]. Nelson MJ, Cowling RA, and Seitz SP (1994) Structural characterization of alkyl and peroxyl radicals in solutions of purple lipoxygenase, Biochemistry 33, 4966-4973. [PubMed: 8161558]

[38]. Ivanov I, Heydeck D, Hofheinz K, Roffeis J, O’Donnell VB, Kuhn H, and Walther M (2010) Molecular enzymology of lipoxygenases, Arch. Biochem. Biophys 503, 161-174. [PubMed: 20801095]

[39]. Sloane D, Leung R, Craik C, and Sigal E (1991) A Primary Determinant for Lipoxygenase Positional Specificity, Nature 354, 149-152. [PubMed: 1944593]

[40]. Ikei KN, Yeung J, Apopa PL, Ceja J, Vesci J, Holman TR, and Holinstat M (2012) Investigations of human platelet-type 12-lipoxygenase: role of lipoxygenase products in platelet activation, J. Lipid Res 53, 2546-2559. [PubMed: 22984144]

[41]. Armstrong M, van Hoorebeke C, Horn T, Deschamps J, Freedman JC, Kalyanaraman C, Jacobson MP, and Holman T (2016) Human 15-LOX-1 active site mutations alter inhibitor binding and decrease potency, Bioorg Med Chem 24, 5380-5387. [PubMed: 27647374]

[42]. Knapp MJ, Rickert K, and Klinman JP (2002) Temperature-dependent isotope effects in soybean lipoxygenase-1: Correlating hydrogen tunneling with protein dynamics, J. Am. Chem. Soc 124, 3865-3874. [PubMed: 11942823]

[43]. Neau DB, Gilbert NC, Bartlett SG, Boeglin W, Brash AR, and Newcomer ME (2009) The 1.85 A structure of an 8R-lipoxygenase suggests a general model for lipoxygenase product specificity, Biochemistry 48, 7906-7915. [PubMed: 19594169]

[44]. Xu S, Mueser TC, Marnett LJ, and Funk MO Jr. (2012) Crystal structure of 12-lipoxygenase catalytic-domain-inhibitor complex identifies a substrate-binding channel for catalysis, Structure 20, 1490-1497. [PubMed: 22795085] 


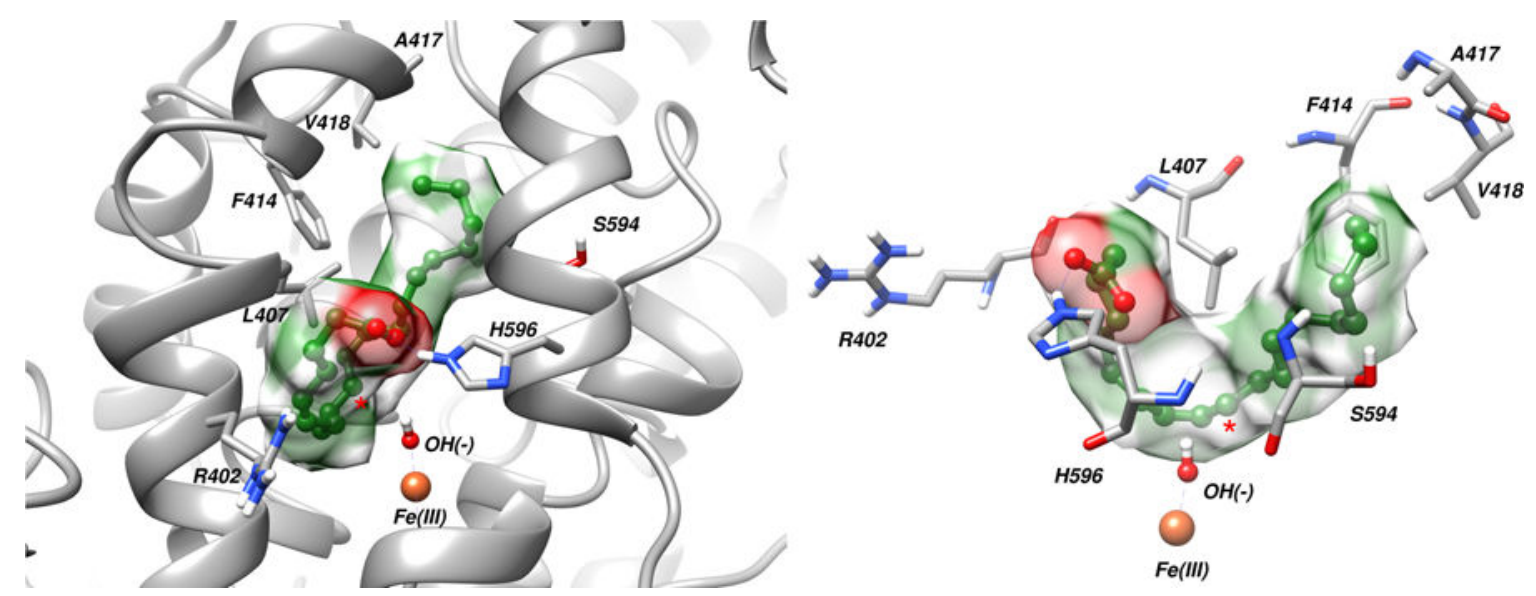

Figure 1.

Two representations of the predicted binding mode of the AA C-10 carbanion intermediate in the hALOX12 wild-type enzyme active site. The negatively charged carbanion C10 is identified with a star $\left(^{*}\right)$. H596 is predicted to form a hydrogen bond to the carboxylate group of the fatty acid. The AA carbanion intermediate is shown in a ball-and-stick and surface representations. The hydroxide ion is shown in a ball-and-stick representation while the key residues that are mutated in this study are shown in a stick representation. Carbon atoms of AA and the protein are shown in grey and green, respectively. Iron is depicted as an orange sphere. Nitrogen, oxygen atom hydrogen atoms are shown in blue, red and white colors, respectively. 


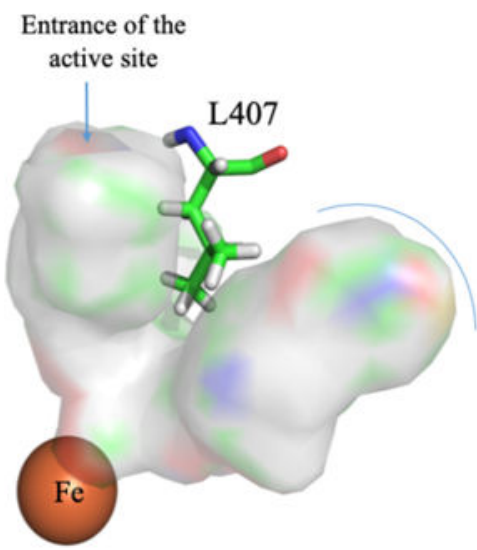

Wt-12-LOX

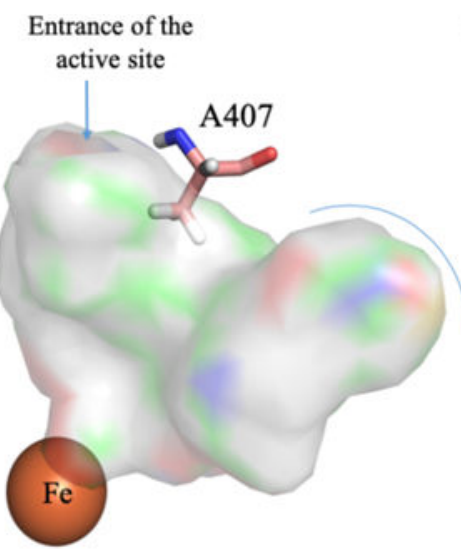

L407A

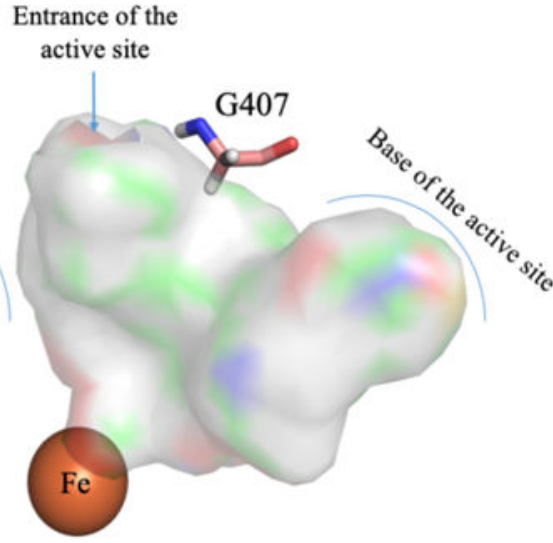

L407G

Figure 2.

Relative cavity shapes and sizes of wt-hALOX12, L407A and L407G. Pymol was used in the generation of this figure. 


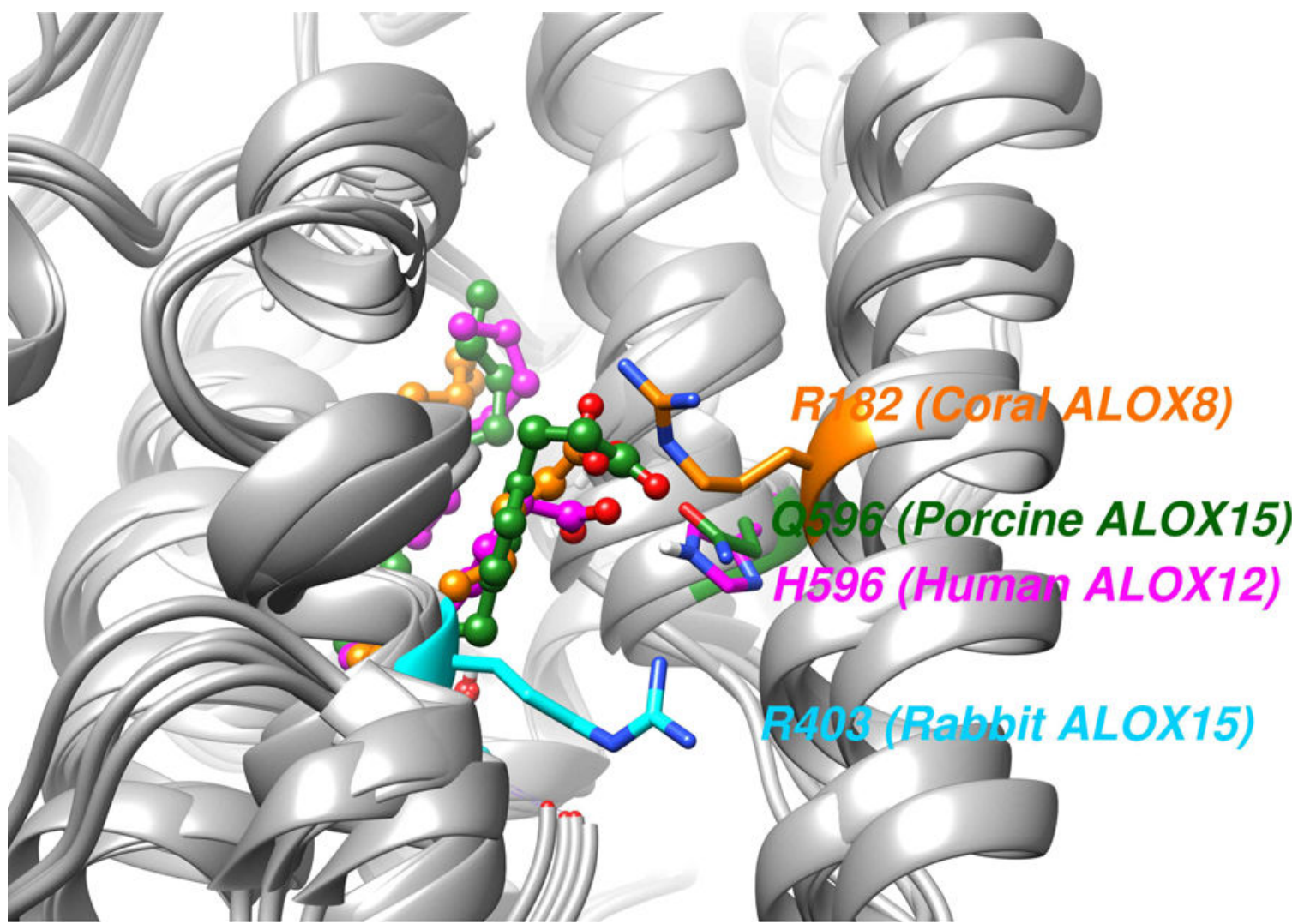

Figure 3.

Superimposed crystal structures of lipoxygenases with differing product specificity: cALOX8 (PDB ID 4qwt) co-crystallized with AA (orange), rALOX15 (PDB ID 2p0m) (cyan), pALOX15 (PDB ID 3rde) co-crystallized with a substrate analog inhibitor (green), and AA carbanion-intermediate docked model of hALOX12 from this work (magenta). The side chains of R182 from helix a 2 of cALOX8, Q596 from helix a 21 of pALOX15, H596 from helix a 11 of hALOX12, and R403 from helix a 11 of rALOX15, which co-ordinate with the carboxylate group of the fatty acid, are also shown. Nitrogen, oxygen and hydrogen atoms are shown in blue, red and white colors respectively. 


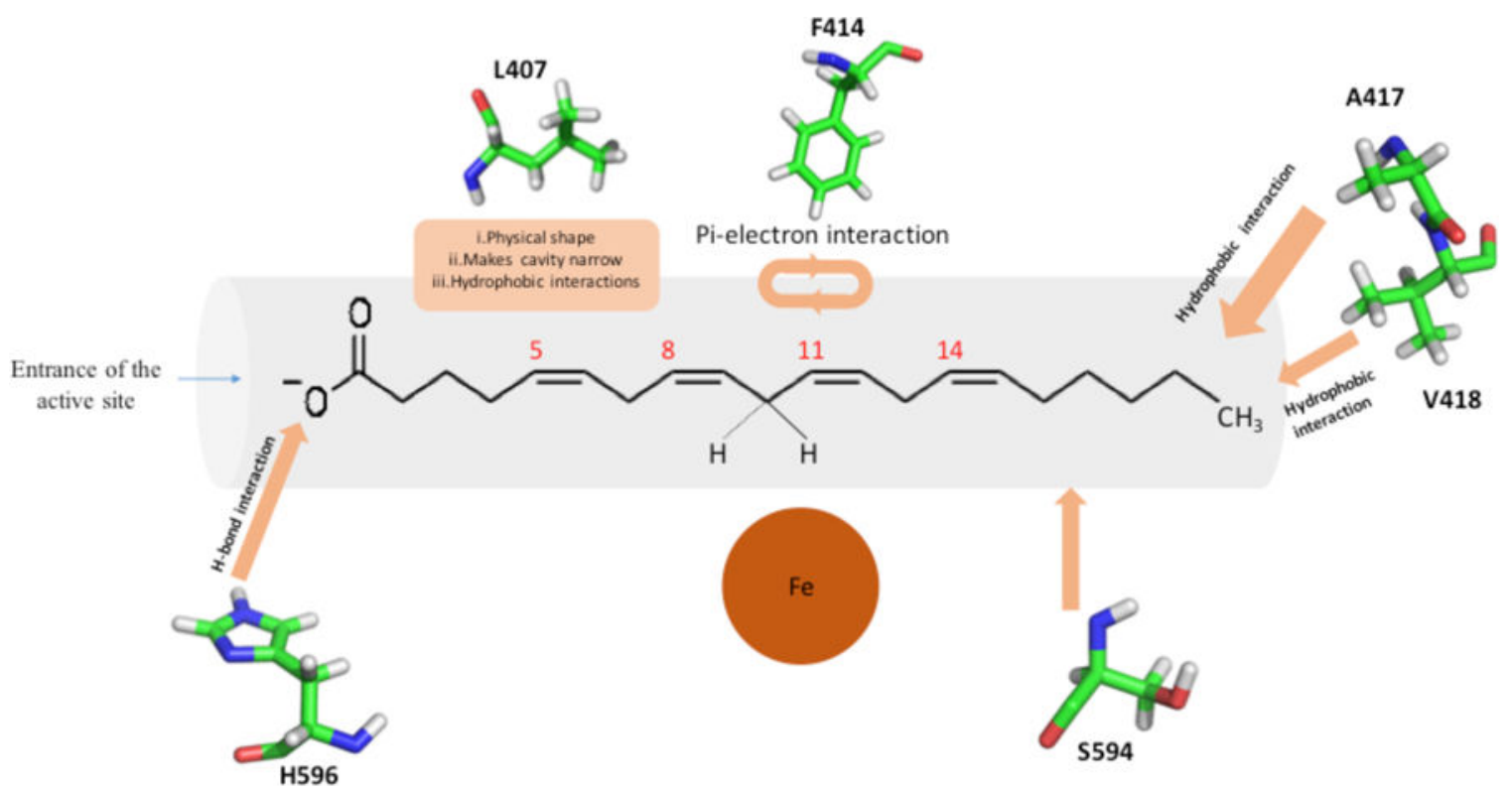

Figure 4.

Schematic diagram of the proposed binding determinants of human 12-Lipoxygenase. The active site is depicted to be straight instead of boot-shaped for easy understanding. This schematic approximates the position of the amino acids relative to carbon in arachidonic acid. 
Table 1.

Mutagenesis experiments to interrogate binding of the carboxylate end of fatty acid substrates with hALOX12. Steady state kinetics measurements with AA and DHA reveal only relatively small differences in the catalytic efficiency between wt-hALOX12 and the 2 mutants. Major changes in the positional specificity are observed for H596L but not R402L, with AA.

\begin{tabular}{|cccccc|}
\hline Substrate & & \multicolumn{2}{c}{ Arachidonic acid } & \multicolumn{2}{c|}{ Docosahexaenoic acid } \\
Enzymes & wt-hALOX12 & H596L & R402L & wt-hALOX12 & R402L \\
$\mathrm{K}_{\mathrm{M}}(\mu \mathrm{M})$ & $1.9 \pm 0.2$ & $1.3 \pm 0.4$ & $0.89 \pm 0.09$ & $0.59 \pm 0.1$ & $1.5 \pm 0.5$ \\
$\mathrm{~K}_{\mathrm{cat}}\left(\mathrm{s}^{-1}\right)$ & $28 \pm 0.9$ & $8.6 \pm 0.7$ & $5.6 \pm 0.1$ & $4.5 \pm 0.1$ & $5.6 \pm 0.5$ \\
$\mathrm{~K}_{\mathrm{cat}} / \mathrm{K}_{\mathrm{M}}(\mu \mathrm{M})^{-1} \mathrm{~s}^{-1}$ & $14 \pm 0.9$ & $6.3 \pm 0.4$ & $6.3 \pm 0.5$ & $7.6 \pm 1.5$ & $3.8 \pm 1$ \\
Product profile (12-HETE:15-HETE:11-HETE) & $100: 0: 0$ & $73: 22: 5$ & $100: 0: 0$ & $29: 71^{a}$ & $37: 63^{a}$ \\
\hline
\end{tabular}

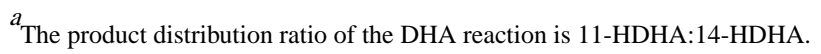


Table 2.

Steady state kinetics and product profile of wt-hALOX12 and its mutants with arachidonic acid to test the aromatic interactions between these residues and the substrate.

\begin{tabular}{|cccccc|}
\hline \multirow{2}{*}{ Enzymes } & \multirow{2}{*}{$\mathbf{K}_{\mathbf{M}}(\boldsymbol{\mu M})$} & $\mathbf{k}_{\text {cat }}\left(\mathbf{s}^{\mathbf{- 1}}\right)$ & $\mathbf{k}_{\mathbf{c a t}} / \mathbf{K}_{\mathbf{M}}(\boldsymbol{\mu M})^{-\mathbf{1}} \mathbf{s}^{\mathbf{- 1}}$ & \multicolumn{2}{c|}{ Products (\%) } \\
& & & & $12-\mathrm{HETE}$ & $\mathbf{1 5 - H E T E}$ \\
wt-hALOX12 & $1.9 \pm 0.2$ & $28 \pm 0.9$ & $14 \pm 0.9$ & $100 \pm 5$ & 0 \\
F414L & $1.4 \pm 0.2$ & $1.7 \pm 0.06$ & $1.2 \pm 0.1$ & $97 \pm 4$ & $3 \pm 1$ \\
F414W & $4.0 \pm 0.3$ & $16 \pm 0.6$ & $4.0 \pm 0.2$ & $100 \pm 5$ & 0 \\
\hline
\end{tabular}


Table 3.

Steady state kinetics and product profile of wt-hALOX12 and its two 414 mutants with mead acid to examine the interacting double bond of the substrate with F414.

\begin{tabular}{|cccccc|}
\hline Enzymes & $\mathbf{K}_{\mathbf{M}}(\boldsymbol{\mu M})$ & $\mathbf{k}_{\mathrm{cat}}\left(\mathbf{s}^{\mathbf{- 1}}\right)$ & $\mathbf{k}_{\mathrm{cat}} / \mathbf{K}_{\mathbf{M}}(\boldsymbol{\mu M})^{-\mathbf{1}} \mathbf{s}^{\mathbf{- 1}}$ & \multicolumn{2}{c|}{ Products (\%) } \\
& & & & $12-\mathrm{HETE}$ & $\mathbf{1 5 - H E T E}$ \\
Wt-hALOX12 & $1.3 \pm 0.09$ & $1.5 \pm 0.03$ & $1.2 \pm 0.06$ & $100 \pm 5$ & 0 \\
F414L & $1.8 \pm 0.5$ & $0.60 \pm 0.05$ & $0.33 \pm 0.07$ & $100 \pm 5$ & 0 \\
F414W & $1.9 \pm 0.2$ & $8.5 \pm 0.2$ & $4.5 \pm 0.3$ & $100 \pm 5$ & 0 \\
\hline
\end{tabular}


Table 4.

Steady state kinetics of mutant enzymes with shallower and narrower cavity.

\begin{tabular}{|cccccc|}
\hline Enzymes & wt-hALOX12 & A417I & V418M & A417I/V418M & A417I/V418M/S594T \\
$\mathrm{K}_{\mathrm{M}}(\mu \mathrm{M})$ & $1.9 \pm 0.2$ & $0.86 \pm 0.1$ & $3.2 \pm 0.6$ & $0.56 \pm 0.1$ & $0.52 \pm 0.1$ \\
$\mathrm{k}_{\mathrm{cat}}\left(\mathrm{s}^{-1}\right)$ & $28 \pm 0.9$ & $15 \pm 0.4$ & $20 \pm 2$ & $34 \pm 1$ & $2.7 \pm 0.09$ \\
$\mathrm{k}_{\mathrm{cat}} / \mathrm{K}_{\mathrm{M}}(\mu \mathrm{M})^{-1} \mathrm{~s}^{-1}$ & $14 \pm 0.9$ & $18 \pm 2$ & $6.3 \pm 0.7$ & $59 \pm 10$ & $5.1 \pm 0.9$ \\
Product profile (12-HETE:15-HETE:11-HETE) & $100: 0: 0$ & $85: 15: 0$ & $100: 0: 0$ & $75.5: 24: 0.5$ & $56: 46: 0$ \\
\hline
\end{tabular}


Table 5.

Steady-state kinetics of the wt-hALOX12 and A417I/V418M mutant enzyme with linoleic acid (LA), gamma linoleic acid (GLA), and eicosadienoic acid (EDA). The cartoons in the table are depicting how the double mutant restricts the depth of the substrate by two carbons. (NA = No activity above the detection limit).

\begin{tabular}{|c|c|c|c|c|c|c|}
\hline \multirow{2}{*}{$\frac{\text { Substrate }}{\text { Enzymes }}$} & \multicolumn{2}{|c|}{ LA } & \multicolumn{2}{|c|}{ GLA } & \multicolumn{2}{|c|}{ EDA } \\
\hline & wt-hALOX12 & A417I/V418M & wt-hALOX12 & A417I/V418M & wt-hALOX12 & A417I/V418M \\
\hline $\mathrm{K}_{\mathrm{M}}(\mu \mathrm{M})$ & NA & $2.8 \pm 0.5$ & $4.1 \pm 0.9$ & $4.7 \pm 0.2$ & NA & $1.7 \pm 0.6$ \\
\hline $\mathrm{K}_{\text {cat }}\left(\mathrm{s}^{-1}\right)$ & NA & $3.8 \pm 0.3$ & $0.34 \pm 0.03$ & $12 \pm 0.3$ & NA & $2.5 \pm 0.4$ \\
\hline $\begin{array}{c}\mathrm{K}_{\mathrm{cat}} / \mathrm{K}_{\mathrm{M}} \\
(\mu \mathrm{M})^{-1} \mathrm{~s}^{-1}\end{array}$ & NA & $1.4 \pm 0.2$ & $0.08 \pm 0.01$ & $2.6 \pm 0.1$ & NA & $1.4 \pm 0.3$ \\
\hline $\begin{array}{l}\text { Major } \\
\text { Product }\end{array}$ & NA & 13-HpODE & 13-HpOTrE & 13-HpOTrE & NA & 15-HpEDA \\
\hline
\end{tabular}


Table 6.

Steady - state kinetics parameters of wt-hALOX12 and the mutants that makes the binding cavity wider (L407A, L407G) and wider as well as smaller (L407G/A417I/V418M).

\begin{tabular}{|c|c|c|c|c|c|c|}
\hline \multirow{2}{*}{ Protein } & \multirow{2}{*}{$\mathbf{K}_{\mathbf{M}}(\mathbf{u M})(\mathbf{A A})$} & \multirow{2}{*}{$\mathbf{k}_{\text {cat }}\left(\mathbf{s}^{-1}\right)(\mathbf{A A})$} & \multirow{2}{*}{$\mathbf{k}_{\mathrm{cat}} / \mathbf{K}_{\mathrm{M}}(\mathbf{u M})^{-1} \mathrm{~s}^{-1}(\mathbf{A A})$} & \multicolumn{3}{|c|}{ Products with AA (\%) } \\
\hline & & & & 12HрETE & 11HpETE & 15HрETE \\
\hline Wt-hALOX12 & $1.9 \pm 0.2$ & $28 \pm 0.9$ & $14 \pm 0.9$ & $100 \pm 5$ & 0 & 0 \\
\hline L407A & $2.4 \pm 0.2$ & $0.28 \pm 0.01$ & $0.12 \pm 0.01$ & $91 \pm 4$ & $6 \pm 1$ & $3 \pm 1$ \\
\hline L407G & $1.8 \pm 0.3$ & $0.22 \pm 0.01$ & $0.12 \pm 0.02$ & $60 \pm 3$ & $19 \pm 2$ & $21 \pm 2$ \\
\hline L407G/A417I/V418M & $1.5 \pm 0.3$ & $0.19 \pm 0.01$ & $0.13 \pm 0.02$ & $66 \pm 3$ & $3 \pm 1$ & $31 \pm 2$ \\
\hline
\end{tabular}

Biochemistry. Author manuscript; available in PMC 2020 June 04. 